\title{
REPRESENTACIONES SOCIALES SOBRE CALIDAD Y EQUIDAD EN EDUCACIÓN
} SOCIAL REPRESENTATIONS ABOUT QUALITY AND EQUITY IN EDUCATION

\author{
SUSANA ARANCIBIA CARVAJAL $1_{i}$ KAREN AYMA CHAMBERS ${ }_{2}$ CINTHIA CIFUENTES FERNÁNDEZ ${ }_{3}$; NEDIELKA YÁÑEZ \\ ZUVIC $_{4}$ \\ (UNIVERSIDAD CATÓLICA DEL NORTE, Antofagasta, Chile)
}

\begin{abstract}
RESUMEN
Estudio analítico/relacional que, a partir del discurso de estudiantes de pedagogía general básica de universidades, reconstruye las representaciones sociales sobre calidad y equidad de educación. Se utiliza una metodología cualitativa, aplicando entrevistas individuales y grupales. De los resultados se destaca que calidad y equidad en educación está estrechamente vinculadas, repercutiendo en la formación integral del estudiantado. La formación docente sería deficiente sin cumplir con las demandas actuales del contexto nacional y regional: no se les enseñarían estrategias pedagógicas; se les insta a situarse desde un enfoque constructivas, pese a que en las clases recibidas se utilizan casi exclusivamente técnicas incompatibles con este enfoque. Ante este escenario, es la vocación la que moviliza a los estudiantes para complementar su formación, en base al compromiso que tienen con su rol dentro de la sociedad.
\end{abstract}

PALABRAS CLAVE: Educación; calidad; equidad; formación docente.

\section{ABSTRACT}

This analytical/relational study is based on statements made by university students of primary education. It reconstructs their pre-conceptions about quality and equity in education. The qualitative methodology included both individual and group interviews. The results highlight that quality and equity in education are closely linked, and that this link has an impact upon the students' integral formation. Teacher training would be deficient if it failed to meet the current demands of the national and regional contexts: if students are not taught teaching strategies, they are urged to situate themselves in a constructive approach, despite the fact that classes they have taken used almost exclusively techniques which are inconsistent with this approach. Given this scenario, it is the vocation that mobilizes students to supplement their training, based on the commitment they have to their social role.

KEY WORDS: Education; quality; equity; teacher training. 


\section{INTRODUCCIÓN}

El concepto de calidad en educación es histórico, ideológico, político y multidimensional (Duk \& Narvarte, 2008). Su conceptualización dependerá de los intereses de quién lo proponga, ya que implica un juicio de valor respecto del tipo de educación, del ideal de persona y de sociedad que se desea (Astorga \& cols., 2007), lo que repercute en las prácticas para alcanzar dichos ideales.

En Chile la calidad en educación se ha vinculado al desarrollo económico (Consejo Asesor Presidencial para la Calidad de la Educación [CAPE], 2006), dentro de un modelo de educación focalizado en la formación de capital humano. Desde esta perspectiva, calidad en educación queda definida por la cantidad de dinero invertido, el número de establecimientos educacionales, de alumnos por área y de profesores (Elías \& Fernández, 2002). Sin embargo, el CAPE planteó la necesidad de adoptar una mirada ligada al desarrollo humano, en la que el derecho a educación de calidad implica generar las:

Oportunidades para cada persona
(cualquiera sea su condición
psicobiológica, económica, de género
u origen étnico) de desarrollar al
máximo posible sus capacidades
cognitivas, sociales, emocionales,
creativas y espirituales y aprender las
virtudes del carácter en el marco del
respeto por otros y del medio
ambiente (p. 87 ).

Astorga y cols. (2007) proponen una definición con cinco dimensiones: educación como un derecho, pertinencia (significativa para cualquier persona, independiente de su cultura, capacidades e intereses), relevancia (satisfacción de necesidades educativas), eficacia (medición de metas) y eficiencia (asignación y distribución de recursos para lograr dichas metas) y, por último, la equidad. Ésta es la más importante, ya que el mejoramiento de la calidad en educación debe llegar en igualdad de condiciones a toda la población.

Equidad en educación implica inclusión educativa con igualdad de oportunidades, a fin de disminuir las desigualdades que puedan influir en la educación de una persona, ya sea derivadas de circunstancias económicas, sociales, étnicas, territoriales u otras. Sin embargo, igualdad de oportunidades en el acceso a educación no asegura que ésta sea de calidad.

Las evaluaciones gubernamentales, como el Sistema de Medición de Calidad de la Educación (SIMCE) y la Prueba de Selección Universitaria (PSU), evidencian las disparidades de resultados entre los distintos tipos de establecimientos educacionales (Amar, 2007; MINHDA, 2005). Ello refleja la discordante calidad de los aprendizajes de niños/as y jóvenes de distinto origen socioeconómico (FSP, 2010).

Se ha intentado mejorar la educación implementado una serie de cambios, como por ejemplo, extender a 12 años la enseñanza obligatoria, aumentando la población escolar desde algunas decenas de miles a tres millones y medio de estudiantes. Si bien hoy todos los niños/as pueden asistir a una escuela, la preocupación radica en lo que ocurre en dichas escuelas $y$ en el mayor aprovechamiento de las más de doce mil horas que pasa cada niño frente a un docente (Cabezas \& Claro, 2011).

Los docentes son los actores con mayor influencia en el aprendizaje; actúan como guías, modelos y referentes, en especial en la enseñanza básica. En esta etapa los pedagogos se transformarían en el principal protagonista de la acción educativa, marcando el ritmo inicial de aprendizaje y sentando las bases del mismo (Ascorra \& cols., 2003; Vaillant, 2002). 
Un elemento fundamental de toda política educativa son las medidas implementadas para atraer, desarrollar y formar buenos profesores y fomentar su esfuerzo (CAPE, 2006). Con la finalidad de optimizar la formación inicial, en los últimos años el Estado ha propuesto la prueba INICIA como sistema de evaluación de conocimientos y competencias alcanzados por los egresados de las carreras de pedagogía (Fundación Educación 2020, 2011). Durante el ejercicio de la profesión, se mide el desempeño a través de la Evaluación Docente, la que indaga en el desarrollo profesional y da cuenta de los aspectos logrados (Colegio de Profesores, s.f.).

Adicionalmente, se han generado beneficios para los docentes, como el paulatino aumento de remuneraciones (Organización para la Cooperación y el Desarrollo Económico [OECD], 2009), financiamiento para su formación continua, compensaciones salariales y programas de pasantías nacionales e internacionales, junto a becas de estudio para sus hijos. A esto se suman premios a la excelencia pedagógica y beneficios asociados al desempeño de los establecimientos educativos (Pavéz, 2011). Una de las últimas medidas del MINEDUC es la beca Vocación Profesor. Sin embargo, el impacto de estas medidas sigue siendo bajo, al estar escasamente asociadas a la efectividad del profesor. Además, la baja valoración social de la profesión, una estructura salarial relativamente uniforme y plana, influye directamente en las perspectivas salariales, y en la consecuente desmotivación de estudiantes talentosos por estudiar pedagogía (Cabezas \& Claro, 2011).

Algunas investigaciones han concluido que los educadores perciben pertenecer a una profesión subvalorada por la sociedad (Ávalos, Cavada, Pardo \& Sotomayor, 2010), motivo por el cual se hace relevante analizar cómo estas representaciones afectan la forma en que interpretan su profesión y sus futuras prácticas.

La sociedad del conocimiento requiere no sólo profesionales técnicamente preparados, sino además con habilidades personales para conducir procesos de enseñanza y de aprendizaje (Vaillant, 2002; Schiefelbein \& cols., 2005). Los futuros docentes deben desarrollar competencias para participar en la vida pública y progresar hacia un enfoque centrado en la educación continua, competencias que debieran adquirir durante su formación inicial. Sin embargo, según González (2010), el aprendizaje en las universidades chilenas, estaría basado en la memorización de contenidos, el aprendizaje individual y se evaluaría mediante pruebas tradicionales que fomentan la reproducción de contenidos. En términos de calidad, para la OECD (2010) la docencia universitaria de pregrado es el ámbito más débil, reflejando la relevancia de indagar en la visión que los estudiantes de pedagogía tienen acerca de la calidad de su proceso de formación.

La revaloración social de la profesión docente pasa por obtener buenos resultados, mejorando la calidad de los profesores, a través de una constante especialización, el trabajo conjunto con el Estado y la innovación en metodologías acordes a la idiosincrasia del país. En este sentido, comprender el rol docente desde las representaciones sociales de quienes estudian Pedagogía General Básica (PGB), entrega elementos para orientar sus futuras prácticas profesionales hacia un enfoque de calidad.

Indagar en las representación social (RS) de los/as estudiantes de pedagogía, es una forma de acceder a elementos simbólicos en torno a calidad y equidad en educación. El término Representaciones Sociales (RS) propuesto por Moscovici (1961) alude a "una modalidad particular del conocimiento, cuya función es la 
elaboración de comportamientos y la comunicación entre los individuos" (p.17).

El conocimiento implícito de las RS son saberes de sentido común, que se constituyen a partir de las propias experiencias, de informaciones, conocimientos y modelos de pensamiento que se reciben y transmiten mediante tradiciones, educación y comunicación. Así, el conocimiento es socialmente elaborado y colectivo (Moscovici, 1984), referido a objetos compartidos por diferentes grupos que constituyen la realidad (Jodelet, 1986). Considerar las RS permite reconocer modos y procesos de conformación del pensamiento social, por medio de los cuales las personas construyen y a la vez son construidas por la realidad.

El presente estudio se sitúa desde el enfoque procesual de las RS, el que fue desarrollado por Jodelet, comprendiendo al ser humano como productor de sentidos, centrándose en la producción simbólica para acceder al conocimiento (Banchs, 2000). Así, este estudio permite comprender algunas de las ideas que configuran los sistemas de creencias del universo simbólico de la docencia.

En síntesis, el objetivo central es reconstruir las representaciones sociales en torno a calidad y equidad en educación que tienen estudiantes de pedagogía general básica de universidades de la ciudad de Antofagasta. Desde el discurso de los participantes se busca comprender ¿En qué contexto emergen las RS sobre calidad y equidad en educación? y ¿Cuáles son las experiencias, creencias y valoraciones vinculadas a ellas?.

\section{MÉTODO}

\section{Participantes}

Con un muestreo teórico, se establece el arranque muestral, y se selecciona a los participantes conforme a las hipótesis emergentes derivadas del análisis de la información. La homogeneidad está dada por la unidad de análisis: estudiantes de PGB (Briones, 1990). Teniendo a la base las RS, la selección de participantes se realiza mediante la estrategia de variación máxima (Trigwell \& Ashwin, 2006). Esta consiste en seleccionar deliberadamente un grupo heterogéneo de participantes (Denzin \& Lincoln, 1998), definido según los siguientes criterios de inclusión: hombres y mujeres, que estudian carreras de PGB de una universidad estatal y dos universidades privadas de la ciudad de Antofagasta. El número total de participantes, determinado por el punto en el que se logra la saturación de la información fue de 16 estudiantes.

\section{Procedimiento general}

Para convocar a los participantes se contactó a informantes clave, quienes proporcionaron la información para comunicarse con estudiantes que cumplían los criterios de inclusión propuestos. Después de que cada estudiante accedió a colaborar, se definieron fechas para entrevistas individuales y grupales, las que se efectuaron en dependencias de las Universidades privadas y en un Centro de Educación y Capacitación.

\section{Criterios de validación}

Para asegurar rigurosidad investigativa se utilizan como criterios de validación: triangulación por investigadoras, triangulación por expertos, exhaustividad, triangulación por técnica, criterios éticos y saturación de información (Valles, 2003).

Procedimiento de producción y análisis de información

Como técnicas de producción de información se utilizan 2 entrevistas individuales y 3 grupales. Las entrevistas individuales se aplican a informantes clave para complementar el guión temático y asegurar la pertinencia de éste. Las 
entrevistas grupales estuvieron guiadas por los siguientes ejes temáticos fueron: calidad y equidad en la educación, formación y rol docente.

El análisis de información se realizó utilizando herramientas propuestas por la Teoría Fundamentada (Glasser \& Strauss, 1967), la cual permite tanto la descripción como la comprensión del fenómeno estudiado. El análisis de la información se efectuó a través de un análisis descriptivo (codificación abierta) y un análisis relacional (codificación axial y selectiva) (Araya, 2002), concluyendo en una aproximación a la codificación selectiva, con la construcción de un modelo comprensivo general que permita articular los contenidos esenciales de las RS (Arancibia, 2008).

\section{RESULTADOS Y DISCUSIÓN}

A partir de los discursos de los/as estudiantes de PGB, las RS emergen agrupadas en cuatro dimensiones principales: Equidad y Calidad en educación, Formación y Rol Docente. Dichas RS se han construido en el contexto político nacional actual, marcado por la agudización de una perspectiva centrada en el capitalismo, que avala explícitamente el lucro y considera la educación un bien de consumo. En este escenario, resurge el descontento y cuestionamiento del modelo educacional vigente, traducido en un movimiento social liderado por los estudiantes, quienes exigen mejoras en el sistema educativo. Las RS de los estudiantes respecto a equidad, calidad en educación y rol docente están ligadas transversalmente a su trayectoria vital. Durante el proceso de socialización primaria la persona es influenciada por sus referentes, según la situación que ocupan dentro de la estructura social y también en virtud de sus idiosincrasias, biográficamente arraigadas (Berger \& Luckmann, 1967).

Las experiencias vividas por los futuros docentes contribuyen a la configuración de sus RS, lo que trasciende e impacta sus procesos de formación actuales. Se considera además las ideologías políticas a la base de cada individuo, junto a las demandas que le hace la sociedad, ya que dependiendo de éstas los participantes cumplen distintos roles, como el ser padres, trabajadores y estudiantes.

Equidad en educación: $A$ otros enseñaron secretos que a ti no.

Una carrera universitaria tiene un costo mensual mayor al sueldo mínimo, lo que refleja parte de la inequidad en el acceso a la educación superior, al ser las condiciones socioeconómicas el factor principal en la adquisición y distribución desigual de la educación (FSP, 2010).

Las diferencias en el tipo de financiamiento que reciben las unidades educativas de enseñanza básica y media, marcan desigualdades en el acceso a la educación superior, ya que las posibilidades de elegir libremente un establecimiento dependen del NSE de las familias. Así, los estudiantes de bajo NSE son los más perjudicados, al estar limitados a asistir a colegios municipales que cuentan con escuálidos presupuestos y escasos materiales.

El1UA:"un trabajador con un sueldo mínimo de 180. Tiene pa' la escuela pública no más" (\$102).

En cambio los estudiantes de NSE alto pueden escoger el establecimiento que deseen, contribuyendo con recursos propios que permiten complementar los materiales de los colegios. En consecuencia, los docentes no se restringen en el aspecto pedagógico pudiendo utilizar las herramientas que consideren pertinentes y necesarias:

E1IUA:"hay unos alumnos que tienen todos los materiales, otros no. Por ejemplo allá en el San Luis tengo todo, 
tengo data, tengo metalófono, tengo material para todos los niños, porque los papás se los compran. Pero yo sé que si voy a trabajar a una escuela municipal no voy a poder aplicar el método en colores que yo hago [...] porque no va haber método para todos los niños" (\30).

La equidad estaría ligada a la calidad en educación, ya que los recursos y condiciones de aprendizaje difieren según el tipo de centros educativo. Esto se evidencia en la medición de calidad en educación recibida, revelando sobretodo diferencias ligadas al NSE del estudiantado:

E1GUA:"la PSU y el SIMCE lo único que refleja, ni siquiera que la escuela es mala, sino que el niño viene de un barrio pobre" (\$120).

Es evidente la segregación de estudiantes, quienes al recibir baja calidad educativa y no tener posibilidades de complementarla, no tienen más alternativa que ingresar al mundo laboral o estudiar en una universidad privada, donde el único requisito es tener los recursos monetarios necesarios. Sin embargo, como plantea Román (2011), los estudiantes con menos recursos no obtienen buenos resultados en la PSU y no logran entrar a universidades tradicionales, ingresando a universidades privadas recurriendo al endeudamiento.

E1IUA:"A unos niños los forman así bacán pa' que den la PSU y entren a la universidad. $Y$ a otros no pu. Los niños saben que no van a dar la PSU, porque saben que no van a tener plata pa' pagar la U." (†98).

En este sentido, la equidad educativa en Chile no es tal, ya que es el NSE uno de los factores que incide en las condiciones en que se da el aprendizaje. Por lo tanto, considerando las RS de los/as participantes y la definición del CAPE (2006), en nuestro país la educación estaría definida desde la inequidad en el acceso a educación y los recursos. Para los participantes, los exámenes de admisión y selección exigidos por colegios subvencionados y particulares, son la evidencia más clara de las desigualdades en educación. Esto hace que los niveles de desempeño se relacionen con el establecimiento de procedencia, y contribuye a que los estudiantes se hagan conscientes de la injusticia de la cual son parte.

E2GUA:"me acuerdo que me fue súper bien en octavo [...] me dijeron "vamos a dar una prueba al XXX" y di la prueba y no, yo me quería morir... me quedé hasta el final con una compañera porque, un montón de materia que yo no tenía idea [...] me sentía tan mal, tan como con una injusticia, imagínate en octavo año ya sentirte que, que no hay para ti lo que otros tienen, o sea, ver niños que en 15 minutos o media hora ya entregaban y se iban y tú ahí dando bote...y uno, ahi yo me sentí... mal"(\18).

Por lo tanto, para los participantes, mientras se divida a los colegios por su sistema de financiamiento, estas desigualdades no desaparecerán. Frente a esto, una de las propuestas es la gratuidad de la educación. Al ser el NSE del estudiantado irrelevante, se educaría en igualdad de condiciones, sin brechas relacionadas con los recursos que posee cada familia:

E1IUA:"debería ser gratuita, debería tener todo, todo, todo el abanico de posibilidades de que tú de verdad te desarrolli como persona" (П72).E6GUPV:"En cambio, si a todos es igual, sea de la condición económica que sea... eh, va a ser la educación igual para todos. Yo creo que ahi está la clave" (†94). 
En este sentido, se relaciona erróneamente gratuidad con calidad en educación, ya que esta condición no implicaría necesariamente el cumplimiento de los estándares de calidad. Gratuidad debe vincularse principalmente a equidad, lo cual garantizaría el acceso, mejorando la inclusión educativa, enfocándose no sólo en cuánto dinero se invierte en educación, sino en cómo se gestiona.

Calidad de la educación: Del saber, del hacer y del ser.

Para los estudiantes de las universidades privadas, calidad en educación implica la entrega de contenidos acordes a un determinado nivel escolar. Para ellos la educación tendría un carácter instrumental, siendo un medio para alcanzar metas curriculares. Dicha definición homologa la educación con el aprendizaje de contenidos, resaltando principalmente el papel que tienen los docentes dentro de este proceso, atribuyéndoles toda la responsabilidad sobre éste:

E2GUDM: "Yo creo que calidad de
educación es que todos los niños
aprendan [...] lo que nosotros
llamamos los "aprendizajes
esperados"(†78).

Los estudiantes de la universidad estatal reafirman el papel protagónico de los docentes, sin embargo, desde su perspectiva la educación no sólo implicaría la adquisición de contenidos, sino también los valores necesarios para el desarrollo de una persona dentro de la sociedad. Esta definición, de carácter integral, resalta la importancia de la adquisición de destrezas interpersonales y no las netamente académicas. Por lo tanto, una educación de calidad requiere docentes preocupados no sólo de los procesos de enseñanza y de aprendizaje de contenidos, sino de formar seres humanos integrales: por ejemplo, los contenidos puramente las materias [...] que aprendan a escribir y a leer, que lo aprendan bien, pero que también aprendan a ser personas [...] los niños tienen que aprender... no sé, solidaridad, el apoyo, que vive en una sociedad, que aprendan a reclamar sus derechos y a cumplir sus deberes." (\134).

Cada actor, dependiendo de su visión política e ideológica, tiene distintas formas de definir calidad de educación (Astorga \& cols., 2007). Los estudiantes de pedagogía de las universidades privadas ligan el concepto a una visión económica, según la cual el estudiante debe formarse como capital humano. Por su parte, los estudiantes de la universidad estatal se enfocan en los derechos fundamentales, visión enmarcada en la educación para el desarrollo humano (CAPE, 2006), según la cual educación de calidad implica la formación de personas desde una perspectiva holística, aprovechando sus potencialidades y en vistas a contribuir al bien común. Así, considerando las dimensiones del concepto calidad de Astorga \& cols. (2007), los estudiantes de las instituciones privadas sólo contemplan las dimensiones de eficacia y eficiencia, mientras que los estudiantes de la universidad estatal, además de éstas, consideran el respeto a los derechos, la pertinencia y la equidad. Estas diferencias podrían repercutir en sus prácticas futuras, en los objetivos, metodologías y en el sentido atribuido a su quehacer.

Para los/as participantes, dentro de la sociedad existen distintos actores, con diferentes grados de compromiso con la calidad de educación. Uno de los principales serían los docentes, ya que son los responsables directos de guiar y apoyar el proceso de aprendizaje, considerando no sólo su desarrollo académico sino que además el área relacional: 
E1GUA: "el rol que tiene el profesor en la sociedad: como el que guía a los niños, como lo veo yo, guía a los niños en cuanto a la forma de generar sus propios conocimientos, como a encontrar la mejor forma para ellos aprender" (\3).

E2GUA: "primero obviamente está la responsabilidad del profesor en hacer bien las clases o ser, no sé si la palabra sea cariñoso, pero entender a sus alumnos, congeniar con ellos, contextualizarse con los alumnos" (\$144).

Situándose desde el rol de futuros docentes, los/as participantes visualizan al estudiante como el actor principal dentro de la educación, ya que son los principales afectados con cualquier medida tomada en esta materia. A pesar de que los futuros profesores consideran explícitamente al estudiante como el actor principal del proceso educativo, implícitamente siguen considerándolos pasivos, dependientes de las prácticas docentes. Tal como lo plantea Vaillant (2002), como serán profesores de educación básica, su labor implicará sentar las bases del aprendizaje, marcar el ritmo y desarrollar programas adecuados acorde a las características de sus estudiantes, aunque las relaciones pedagógicas se basarían en la autoridad desigual.

E1GUDM:"Es que el estudiante es el actor principal en todo esto, sin el estudiante nosotros no seríamos nada, porque por ellos nosotros tratamos de mejorar, de adquirir nuevas estrategias para enseñar" ( $(91)$.

Asimismo, ligan el rol docente de educación básica con el rol materno, debido a la etapa evolutiva en que se encuentran los estudiantes. Además, son mujeres quienes postulan en mayor proporción a pedagogía general básica, probablemente siguiendo el mandato social que las sitúa como las encargadas de asumir funciones de cuidadoras. Como los estereotipos de género les atribuyen funciones de sociabilidad y simpatía interpersonal, la educación se ve feminizada, a raíz de la presión social que mandata que sólo las mujeres cumplan el rol de educar y cuidar a los niños (Páez \& cols., 2003). Pese a los intentos por incluir una perspectiva de género en educación, los estereotipos siguen presentes en carreras ligadas al trabajo con niños.

E1IUA:"la educación también está feminizada... son puras profesoras, son mujeres y sobre todo en la básica, por el mismo rol que la mujer tiene que estar ligada a los niños, a la crianza." (\268).

Los padres son identificados por los participantes, como actores implicados en la calidad de educación. Es indispensable que la visión del profesor considere otros actores dentro del proceso de aprendizaje, ya que la familia del estudiante actúa como referente en el proceso de socialización primaria, por lo que el trabajo conjunto permitiría que ambos actores se sincronizaran a favor de una formación integral del estudiante:

E2GUDM: "la primera escuela que tienen los niños es la familia" (†89).

Dentro de un establecimiento existen otros actores, como directivos $y$ paradocentes, quienes tienen por misión acompañar y asesorar el proceso educativo. Su función implica la gestión educativa y ámbitos que van más allá de la formación académica, como el sistema relacional. Por tanto, la educación es un proceso complejo que involucra no sólo la formación dentro del aula, sino que también la gestión organizacional de cada establecimiento:

E1GUDM:"Yo creo que a la gestión. Si alguien de arriba, en el área de educación, por ejemplo el director. Entonces el debería decir que se 
trabaje de tal manera, que sea mucho más significativa" (\43).

A nivel macro, los participantes consideran al Estado como actor responsable de asegurar la calidad y equidad de educación, siendo su deber otorgar los recursos necesarios para que los procesos educativos se desarrollen adecuadamente, además de entregar lineamientos de contenidos y metodologías a utilizar en los establecimientos educacionales del país. Los diferentes establecimientos no reciben los mismos estándares de calidad, favoreciendo a los estratos de NSE alto. Tanto el gobierno actual como los anteriores han intentado mejorar la educación (OECD, 2009), con soluciones que no tienen el impacto suficiente para que ésta cambie, ya que generar nuevas estructuras y renovar materiales no implica que el uso de éstos serán los apropiados ni tampoco que los estudiantes percibirán estos cambios:

\section{E6GUPV:"El Estado digamos es una parte fundamental para que haya educación. Entrega los recursos y entrega también la forma de trabajo, aunque la metodología uno la pone, pero todo lo que significa planificación y programas, etc., el Estado tiene que ponerlo" (\87).}

Es importante destacar el trabajo conjunto por parte de los actores educativos, incluyendo a la sociedad en general, ya que es una temática que compete a toda la población por el carácter transversal en la vida del sujeto como ser social. Las movilizaciones estudiantiles del 2011 han reposicionado la importancia de la educación dentro de la sociedad. Asimismo, los estudiantes tienen posiciones polarizadas en torno a la participación. Por un lado, los entrevistados de universidades privadas manifiestan su rechazo a las movilizaciones por considerar que la calidad de la educación es una utopía y que la gratuidad no es viable, proponiendo que con el aumento de las becas bastaría. Este discurso estaría sesgado por el contexto en el cual estudian, ya que este tipo de universidades avala el lucro, promueve la educación como un bien de mercado y prohíbe toda participación que no se remita a lo netamente académico.

E1GUST:"no sé si es que la educación fuese gratis, pero que te dieran más instancias para poder estudiar" (\209). E2GUPV:"En el fondo, para mí calidad de la educación es como una utopía, es un sueño" (\$51).

E1GUST:"Por eso mismo, no se van a paro. $Y$ si participas en esas cosas, bueno te dicen po, que estaría peligrando tu matrícula" (\204).

Por otro lado, los estudiantes de la universidad estatal apoyan y participan en las movilizaciones, las que son significadas como motor de cambio social, ya que a partir del cuestionamiento al sistema educativo se ha configurado una nueva forma de ver la sociedad. Se pone en discusión el rol del sector público frente a los derechos sociales y se critica el rol del Estado ligado al mercado. El cuestionamiento del lucro y de la mercantilización de los derechos sociales, como la educación y los servicios públicos, evidencia la indignante concentración de la riqueza, en un contexto de desigualdad económica:

E4GUA:"para mí, qué ha cambiado la
movilización, cambio el rol de cómo se
ve la sociedad, cómo se ve la
educación, ya no se ve como los
pobres tienen que estudiar en un
ghetto y los ricos que estudien ahí en
el San Esteban" (\215).

Estas RS de calidad de educación repercutiría en su quehacer profesional, por lo que un docente que no cree en la factibilidad de mejorar el estado actual de la educación no se comprometería con instancias de cambio, lo que afectaría su 
desempeño en términos de calidad $y$ equidad. En tanto que un docente en formación comprometido con cambios que apunten al mejoramiento de la educación, implicaría que sus prácticas estuvieran enfocadas a éstos.

\section{Formación docente: Aprender a enseñar.}

Desde el doble rol que van adoptando los participantes, a medida que van profundizando los argumentos en torno a calidad de educación, emergen contenidos acerca de su propia formación como futuros docentes. Independiente de la universidad en que cursan sus estudios, consideran que su formación se enfoca principalmente en la adquisición de contenidos específicos del currículum más que en metodologías y desarrollo de competencias pedagógicas específicas para enseñar dichos contenidos.

E2GUA:"Encuentro que ellos deberían habernos enseñado a enseñar y eso yo creo que, cuánto habremos tenido de enseñar a enseñar: ¿un 5\%?"(\34).

Incluso consideran insuficiente la entrega de dichos contenidos, a pesar de que su formación se basa principalmente en ésta. Contrastando esta situación con la definición de calidad que ellos mismos proponen, su formación como futuros docentes no contemplaría su desarrollo integral ni existiría una mayor preocupación por lograr los aprendizajes esperados para su buen desempeño como pedagogos. Este resultado es especialmente relevante, ya que los participantes están inmersos en un proceso dialéctico, donde al no aprender de manera óptima cómo ejercer su labor, es probable que sus futuros estudiantes tampoco se eduquen en calidad.

Para llenar esos vacíos, una de las estrategias utilizadas es la revisión de la literatura escolar utilizada en su propia educación básica. En este sentido, la autogestión y la pro actividad son estrategias que les han permitido suplir estas falencias. Sin embargo, el hecho de utilizar material sin las reformulaciones recientes no es adecuado, dado que implica la desactualización de los contenidos trabajados por docentes y estudiantes, lo que a su vez incide en el desigual bagaje de información que están aprendiendo, y que a futuro desarrollarán en su quehacer profesional.

E1GUA:"Yo creo que la mayoría, recurrió a la autoeducación, por ejemplo si yo me sabía algo yo recurría a un libro o un resumen para ver lo que me pasaron en la básica." (†82).

E1IUA:"Todo lo que yo sé y la capacidad que yo tengo para enseñar ha sido porque yo me he formado aparte, pero en la carrera no te enseñan a enseñar." (\20).

Pese a que la universidad estatal ha reformulado asignaturas dentro de la malla curricular, siguiendo las exigencias que propone el MINEDUC. Los estudiantes visualizan que estos cambios no han sido significativos, reflejando que su formación sigue siendo deficiente, ya que según su mirada, no responden a las necesidades y competencias consideradas necesarias para una formación integral, dejando a los estudiantes con inseguridades a la hora de ingresar al campo laboral.

E4GUA:"Con la nueva reforma a nosotros nos cambiaron algunas asignaturas, le pusieron aprendizaje de la enseñanza de la educación matemática, aprendizaje de la enseñanza del lenguaje $y$ comunicación. Supuestamente con esos nombres iban a cambiar. Nos iban a enseñar a enseñar educación matemática, pero nunca fue así." ( $(35)$.

A futuro puede que estos profesionales no estén lo suficientemente capacitados 
para entregar una educación pertinente a las necesidades de sus estudiantes, ya que las demandas de la sociedad actual requieren profesionales técnicamente preparados y con habilidades personales para conducir procesos de enseñanza (Schiefelbein \& cols., 2005; Vaillant, 2002). Así las formas de enseñar deben adaptarse a las nuevas tecnologías y el contexto en el cual se desenvuelven sus estudiantes, pero si su formación actual aún no logra adaptarse a los contenidos mínimos, ¿cómo podrían desarrollar estas competencias?. Esta falencia evidencia un proceso circular en cuanto a calidad de educación desde estos actores, debido a que la calidad de la educación recibida en su trayectoria vital, podría repercutir en la calidad que impartan a futuro, considerando que todos los participantes provienen de escuelas municipales y subvencionadas, por lo que la calidad de su educación básica y media limita el aprender aspectos más complejos en su formación (FSP, 2010).

El docente necesita al menos cuatro cualidades: capacidad de construir un punto de vista personal, predisposición para la búsqueda, dominio de conocimiento y competencias y espíritu de colaboración (Fullan, 1993 citado en UNESCO, 2006). Sin embargo, la formación que reciben no se condice con el desarrollo de estas cualidades, por lo que los egresados no estarían lo suficientemente preparados para ejercer su profesión.

E1GUA:"Historia es lo que quiero yo y especializarme en algo y hacer clases de eso, porque con esto que tengo no, no... me daría vergüenza salir a hacer clases asi" (\$103).

E4GUA:"me siento: mal. No me dan ganas de estar trabajando el otro año, no me siento capaz" (\$97).

En este sentido, se vislumbra un escenario estático dentro de las políticas públicas para mejorar la formación docente.
A pesar de las renovaciones en las mallas curriculares en pedagogía, los estudiantes no perciben cambios en los contenidos 0 modos de impartir clases. Las formas de enseñanza dentro de las universidades presentan una disociación entre la teoría y la práctica, ya que los docentes intentan transmitir en la teoría una forma ideal de enseñanza, que incluya el paradigma constructivista, aprendizajes significativos y metodologías innovadoras. Sin embargo, la formación de los propios estudiantes se lleva a cabo sin considerar estos elementos. Adicionalmente, los contenidos de algunas asignaturas son considerados irrelevantes, al no ser abordadas de manera aplicada a la carrera.

E4GUA:"Ellos mismos, el
constructivismo, no lo hacen
constructivistamente, de manera
constructiva, lo hacen de manera
conductista, porque a nosotros nos
hacen un traspaso de conocimiento
nos dicen tomen acá esta forma, pero
tiene que hacerla constructivista, pero
tú dices: ¿pero cómo?" ( $\mid 41)$.
E1IUST:"Yo tuve psicología del
aprendizaje, pero era como...
empezaron a explicarnos cómo era el
feto y cómo la evolución y... pero
cómo atender ponte tú a un niño que
tiene problemas, no así. Entonces, yo
creo que faltaron hartas, de parte de
las didáctica, metodología, fue lo que
más faltó"(\80).

Esta metodología es evaluada negativamente por parte de los estudiantes, quienes consideran necesario que los docentes actualicen e innoven la forma de realizar las clases. Esto coincide con los resultados de la OECD (2010):la formación de pregrado es la más débil en términos de calidad. Ello se evidencia en el esfuerzo por tratar de generar los aprendizajes significativos desde el constructivismo, pero con metodologías incorrectas, que no incluyen el desarrollo de competencias pedagógicas de los futuros docentes. 
Asimismo, es importante aumentar las instancias prácticas a modo de complementar la teoría. Esto se liga a lo que propone González (2010), quien revela que las metodologías no son innovadoras, por lo que tampoco se desarrolla esa competencia en los estudiantes, esto podría repercutir en sus futuras prácticas docentes ya que al no ser formados desde esta perspectiva, implicaría dificultades para abandonar la metodología tradicional.

E1UDM:"tú puedes tener mucha teoría, pero cómo lo vas a llevar a la práctica si no te han enseñado todo. Entonces yo considero que lo que esta universidad tiene son buenos contenidos, tiene buenos profesores, que imparten bien las metodologías, pero considero que se debería trabajar más con teoría y práctica a la vez"(†38).

En la formación docente, el aprender a enseñar, es un elemento deficiente en las aulas de los estudiantes de pedagogía, a pesar de que se piensa que estas temáticas debieran ser las principales en cuanto al desarrollo profesional de un futuro docente. Los mismos estudiantes dan cuenta de este vacío; sin embargo, al parecer no existen instancias en que, tanto estudiantes como docentes y directivos, construyan cambios en pro del mejoramiento de la formación. Lo que cubre este vacío, y que se resalta como fortaleza, según el discurso de los estudiantes, son las prácticas profesionales que han tenido desde el primer año de su formación, ya que les ha permitido reafirmar su vocación, evidenciando la realidad del aula. Además, la posibilidad de insertarse en un establecimiento ha contribuido a enriquecer los contenidos y competencias que intenta desarrollar la carrera.

E1IUST:"lo que sí, me...me ayudó mucho a eso y a reafirmar que eso es realmente lo que me gusta fueron las primeras prácticas que la universidad me... me incorporó" (\2).

En este sentido, los aprendizajes en las prácticas profesionales se ligan al refuerzo de la vocación docente, sumada a la pro actividad y la autoformación, lo que podría incidir en el aporte de mejoras concretas en la calidad de educación. Sin embargo, estos elementos figuran casi externos a la formación que entrega cada institución superior. Dentro de esta formación, los estudiantes consideran que las evaluaciones de las prácticas profesionales se basan en tópicos descontextualizados y poco relevantes en relación al fin del proceso de formación.

E3GUA:"En las sala pasan cosas po, el niño se puede enfermar, puede que no haya tomado desayuno, el niño puede que no haya dormido porque le pegaron en la noche o lo violaron. Pasan cosas po y yo tengo que rápidamente pensar y contextualizar $y$ cambiar esto... y a lo mejor cambiar y sacar esto, chao fuera, no me sirve e improvisar en la clase... y no, te ponen mala nota por eso"(\$109).

En este sentido, los estudiantes visualizan que las pautas de evaluación no son suficientemente flexibles para considerar contextos en el que se dan los procesos de enseñanza y de aprendizaje. Posiblemente los docentes de las instituciones superiores se acogen a una planificación inalterable, enfocándose sólo en el cumplimiento de características tradicionales de un docente y no en el proceso y desempeño que se ha dado a lo largo de las prácticas.

En lo que respecta a evaluaciones de calidad docente desarrolladas desde el gobierno, los estudiantes concuerdan en la importancia de éstas, ya que permiten obtener una retroalimentación del quehacer educativo. Sin embargo, critican la actual Prueba INICIA, ya que sólo considera 
contenidos teóricos, excluyendo la capacidad para desenvolverse dentro del aula. Este resultado demuestra que la formación y evaluación de la calidad del docente sólo se mide en relación a los conocimientos que posee, sin considerar las habilidades pedagógicas.

E3GUDM:"a mí me gusta que me evalúen, yo encuentro que eso es lo mejor que se va poder hacer porque así uno ve cómo sale, cómo sale preparada para ya impartir o enseñar. Y también ser evaluada constantemente le va diciendo a uno, cuáles son las falencias o los errores que tienen así seguir mejorando"(\137).

E1GUA:"La Prueba INICIA mide conocimientos, pero no mide la capacidad del profesor para desenvolverse en un aula [...] no es capaz de ver cómo el profesor trata a los alumnos en la clase." (\$127).

No obstante, los resultados no abordan la calidad, desde las conceptualizaciones entregadas por los estudiantes. Con esto se puede evidenciar la visión que tiene el aparato estatal respecto de la educación y su calidad, caracterizándose por la instrumentalización del docente como el portador de contenidos, los cuales deben ser entregados; no hay énfasis en las competencias necesarias para el proceso de esta entrega de información. Esta perspectiva de calidad de educación se relaciona con la que implica sólo la cantidad de profesores por área, de contenidos a entregar, dinero invertido, número de establecimientos educacionales y de alumnos, la que visualiza la educación como desarrollo del capital humano (Elías \& Fernández, 2002), más que como impulso de la auto-realización de las personas y desenvolvimiento en la sociedad.

De esta forma, para obtener un buen rendimiento en la Prueba INICIA, una de las medidas aplicadas por las universidades privadas y que se está implementando en la universidad estatal, es la organización de talleres obligatorios extracurriculares para sus estudiantes. No obstante, la prueba INICIA no cumple el objetivo de evaluar los conocimientos adquiridos a lo largo de la carrera, sino que ofrece una visión sesgada de la formación como consecuencia de la preparación adicional que se adquiere en estas instancias.

\section{E3GUDM:"acá hacen talleres para la Prueba Inicia."(\147). \\ E1IUA:"quieren abrir talleres para estudiar para la Prueba Inicia. Ya están adaptados al sistema” (\$67).}

Según los discursos, tanto las carreras de pedagogía de las distintas universidades, como sus estudiantes adoptan las medidas derivadas del organismo estatal sin cuestionamientos de los beneficios 0 contradicciones que implica. Por ejemplo, la generación de talleres exclusivos para la prueba INICIA para algunos estudiantes implica el reforzamiento de los aprendizajes que han desarrollado a lo largo de su formación y para otros representa las falencias que han tenido. Las universidades son conscientes de este punto, ya que si la formación hubiese sido la adecuada, no sería necesario realizar estos talleres para responder a los objetivos de la evaluación.

En lo que respecta a la evaluación de la calidad docente, los estudiantes consideran necesario que su desempeño sea evaluado, ya que les permite tener una retroalimentación para mejorar su quehacer. Sin embargo, las metodologías utilizadas serían inadecuadas, ya que según la mirada de los estudiantes son manipuladas y descontextualizadas:

E1IUA:"la evaluación docente es una mentira. Hasta la misma, donde nosotros hacemos el seminario... eh, hay una profesora que les hace el portafolio a todos los profesores de la 
escuela, les cobra y lo hace, para que no los echen" (\266).

E2GUA:"estoy peleando un puntaje. Un número te va a decir como profesional cómo eres y con ese número te van a contratar en un lado 0 no te van a contratar en un lado por un número" (\$122).

En este sentido, la medición de la calidad tanto de la formación docente como del profesional, debiera considerar el consenso del concepto de calidad de educación por parte de todos los actores, ya que existen distintas formas de definirla como personas que la definen. Es por ello que muchas veces los actores no coinciden entre sus propuestas, ya que según los intereses de cada actor podrían implicar directamente juicios de valor acerca del tipo de educación que se quiere para formar un ideal de persona y de sociedad (Astorga \& cols., 2007).

\section{Rol docente: Yo soy la educación.}

El rol docente está definido desde la vocación, asociada al deseo de estar en contacto con otros, cumpliendo la función de ayudar a las personas a través de la educación. Esto trae consigo sentimientos positivos relacionados a la satisfacción con su quehacer profesional. De esta forma, el cumplimiento del rol en un docente con vocación, trae consigo satisfacción personal frente al aprendizaje de sus estudiantes.

E4GUA:"me fui a pedagogía como por el perfil de la carrera que era trabajar con gente, la sociedad y ayudar. Siempre me ha gustado trabajar con personas y estar con gente" (\$12).

La forma en que los participantes se representan el rol docente está definida desde la posibilidad de cambiar el futuro de sus estudiantes. A través de la formación que entreguen podrán generar movilidad social y contribuir a su desarrollo integral que les permitirá desempeñarse en la vida cotidiana. Desde esta visión, el estudiante sigue teniendo un rol pasivo frente a la participación activa del docente, donde su responsabilidad iría más allá de los procesos desarrollados en aula, implicando un cambio a nivel social. En este sentido, los estudiantes de PGB consideran que, como agentes de cambio, deben comprometerse con cualquier acción que tenga como objetivo mejorar la educación. Lo cual no debería sólo remitirse al trabajo en el aula, si no también formar parte de acciones sociales que se organicen.

E2GUA:"el profesor cumple un gran rol en la sociedad que es la de hacer escalar a un niño, que con todas esas cosas que va aprendiendo se va sintiendo una persona más íntegra" ( $(16)$.

E1IUA:"Pero yo pienso que si a ti en realidad te interesa la educación y te interesan los alumnos que tení frente a ti, entonces teni que luchar por un cambio, y que los profesores sí somos fundamentales, pero deberíamos entonces nosotros hacer una educación distinta a la que se está entregando hoy" (\$1).

Esta perspectiva no considera la visión sistémica de la educación, ya que se considera al docente como único contribuyente dentro del proceso de aprendizaje. Un aspecto positivo de esta visión es que los empodera para realizar cambios e innovar en futuras prácticas, sin embargo, existen circunstancias que están fuera de su alcance y que incidirán en su quehacer profesional, desgastándolos tanto física como psicológicamente, por ejemplo, estudiantes con necesidades educativas específicas y otras problemáticas psicosociales como la violencia intrafamiliar. Además, existen factores de su profesión que imposibilitan las acciones para contribuir al cambio social de sus estudiantes, como la sobrecarga laboral y el alto número de estudiantes por sala y el 
contexto en el que deben lidiar a diario, sobre todo en escuelas municipales.

E1IUA:"me acuerdo que una niñita, [...] ella tenía un déficit igual muy profundo. No era para estar en una escuela normal, pero estaba ahí y [...] siempre se quedaba dormida porque no comía, siempre andaba con unas ojeras así gigante. Era asi flaquita, chiquitita y se sentaba en el primer puesto así y una vez estaba haciendo la media clase yo. Yo así me esforzaba por hacer una cuestión entretenida y la niña así estaba raja durmiendo así. $Y$ yo le pregunte Susy: ¿por qué tení tanto sueño? Y dijo: no, es que mi mamá no me dejó dormir anoche. Yo le dije: ¿Por qué? ¿Qué pasó? Dijo: no, es que llegó en la noche y estaba así como rara y me empezó a tirar agua helada a la cama, me hablaba ella en su lengua porque igual le costaba como modular" (\$113).

Otro factor que podría desgastar la fuerza de la vocación es la subvaloración social de la profesión. Esta subvaloración se atribuiría principalmente a las malas condiciones laborales, específicamente las bajas remuneraciones. Esto repercute negativamente en la relación estudianteprofesor, ya que a nivel social las carreras se valoran en función del dinero recibido por su grado, siendo los docentes los que reciben menores remuneraciones en comparación a otras profesiones (UNESCO, 2006).

E2GUDM:"Hoy día a nosotras nos dicen: ah, pero tú estás estudiando pedagogía no más, vas a ser profesor no más. [...] Entonces a veces los mismos alumnos dicen: ah no importa, total el profe es el profe no más" (\89).

Finalmente, la vocación surge nuevamente frente a los factores que inciden en la representación del rol como agentes de cambio social.

E1IUA:"(los profesores) te lo dicen con las palabras asi: da lo mismo si te pagan mal, porque tú soy... tú tení una vocación y tení que con eso llegar adelante" (\$34).

El discurso sobre vocación es utilizado como un argumento para sobreponerse a las malas condiciones laborales, derivando en conformismo frente a las condiciones laborales. La gravedad de esta situación recae en que son los docentes de los participantes quienes utilizan este argumento, para formar a pedagogos que centren la responsabilidad del proceso de aprendizaje en su rol, incentivando una mirada lineal sin considerar el aspecto sistémico de la educación ni cuestionar los bajos sueldos asociados a su profesión.

En conclusión, las RS en torno a equidad y calidad en educación de los estudiantes de PGB, emergen en un contexto sociopolítico caracterizado por las desigualdades socioeconómicas, que tienden a segmentar a los estudiantes en diferentes establecimientos. Por esta razón, es que cada vez existen más voces que exigen cambios sustanciales en materias educativas y tributarias. Este contexto incide en la construcción de las RS, ya que los discursos podrían estar matizados por la información que circula en base a la contingencia nacional. Las RS emergen desde dos vertientes, debido al doble rol desde el cual se posicionan los participantes. Por un lado, como estudiantes de PGB y, por otro, como docentes, lo que permite enriquecer su visión de la educación. Esto se relaciona con su trayectoria vital, ya que dependiendo de ésta, la realidad se vivencia desde distintos sectores sociales. Esto trae consigo diferentes experiencias relacionadas al acceso a educación, formación recibida y sus recursos. 
No se puede definir como un sistema de educación de calidad, a uno que no cuenta con equidad para sus estudiantes. La calidad en educación se define desde dos posturas, la primera ligada al desarrollo integral del estudiante, y la segunda a la formación de capital humano. En este sentido, la calidad de la formación que reciben los estudiantes de pedagogía se liga a la segunda definición, ya que sólo contempla contenidos teóricos y excluye aspectos integrales y relacionales. Incluso no considera aspectos esenciales para el proceso de aprendizaje como es el desarrollo de competencias pedagógicas.

En cuanto a calidad y equidad se reconoce el carácter sistémico de la educación, ya que se identifica una gran cantidad de actores implicados en estas temáticas. No obstante, se releva el rol que tiene el docente y se visualiza como el principal responsable de la acción educativa, quien tendría capacidades para realizar cambios sociales desde el aula. En este punto, la vocación tomaría un rol protagónico, visualizando al docente como agente de cambio que contribuye con metas ligadas a la movilidad social.

Una de las propuestas para que se produzca esta movilidad, coherente con las demandas estudiantiles, es un sistema educacional gratuito, donde el acceso a una educación de calidad no estaría mediado por los recursos que posee el estudiante, sino que estarían repartidos equitativamente entre los colegios. Sin embargo, se liga erróneamente gratuidad con calidad en educación, ya que esta condición no implicaría necesariamente el cumplimiento de los estándares de calidad. Gratuidad en educación implicaría la formación de personas en un contexto en el que se visualizaría la educación como un derecho para el desarrollo humano.

Frente a situaciones que incidirían en el desempeño docente como la desigualdad de género, la subvaloración de la profesión y las malas condiciones laborales, la vocación puede ser entendida como la capacidad del docente para revertir esta situación, mientras que, por otro lado, actuaría como conformismo frente a las condiciones laborales.

Las prácticas y la formación recibida serían un intento de formar integralmente a futuros docentes, aunque esto quedaría sólo en un intento, ya que no se lograría aprender a enseñar en términos de calidad. Frente a esto, es necesario efectuar cambios curriculares que contemplen las necesidades del estudiante, los actores involucrados en los procesos de enseñanza y de aprendizaje, con la finalidad de promover una formación docente acorde a las necesidades actuales.

Debido a que en la presente investigación sólo se pudo acceder al discurso de un estudiante, se sugiere que futuras investigaciones consideren el sexo y los roles de género como variables de análisis, en especial si se tiene en cuenta que los varones son una minoría dentro de la profesión. 
REFERENCIAS

Amar, M. (2007). Equidad, calidad y derecho a la educación en Chile: Hacia un nuevo rol del Estado. Biblioteca del Congreso Nacional. Serie Estudios $\mathrm{N}^{\circ}$, p. p.5-12.

Arancibia, S. (2008). Teoría fundamentada en los datos (Grounded Theory): Representación social del liderazgo juvenil. En M. Cárdenas, P. Salinas (Ed.), Métodos de investigación social: Una aproximación desde las estrategias cuantitativas y cualitativas (pp. 383-397). Antofagasta: Universidad Católica del Norte.

Araya, S. (2002). Las representaciones sociales: Ejes teóricos para su discusión. Costa Rica: Facultad Latinoamericana de Ciencias Sociales (FLACSO).

Ascorra, P., Arias, H. \& Graff, C. (2003). La escuela como contexto de contención social y afectiva. Revista Enfoques Educacionales 5(1), 117-135. Extraído el 23 de Julio del 2011 desde FACSO. Acceso:

http://www.facso.uchile.cl/publicaciones lenfoques/07/Ascorra_Arias_Graff_Esc uelaContencionSocialAfectiva.pdf

Astorga, A., Blanco, R., Guadalupe, C., Hevia, R., Nieto, M., Robalino, M., \& Rojas, A. (2007). Educación de Calidad para todos: Un asunto de Derechos Humanos. Buenos Aires, Argentina: UNESCO. Recuperado de http://unesdoc.unesco.org/images/0015 /001502/150272s.pdf

Ávalos, B., Cavada, P. \& Sotomayor, C. (2010). La profesión docente: temas y discusiones en la literatura internacional. Estudios Pedagógicos, 36(1), 235-263.

Banchs, M. (2000). Aproximaciones Procesuales y Estructurales al estudio de las Representaciones Sociales. Peer Reviewed Online Journal. Vol. 9. 3: $1-15$.

Berger, P., Luckmann, T. (1967). La Construcción Social de la Realidad. Buenos Aires: Amorrortu Ediciones.
Briones, G. (1990). Métodos y técnicas e investigación para las ciencias sociales. México D.F.: Editorial Trillas.

Cabezas, V. \& Claro, F. (2011). Valoración social del profesor en Chile: ¿cómo atraer a alumnos talentosos a estudiar pedagogía? Pontificia Universidad Católica de Chile. Facultad de Educación, 6(42). Recuperado de http://politicaspublicas.uc.cl/media/publi caciones/pdf/20110308110825.pdf

Chile, Consejo Asesor Presidencial para la Calidad de la Educación (2006, diciembre). Informe final del Consejo Asesor para la calidad de la educación. Recuperado de http://www.consejoeducacion.cl/articulo s/Informefinal.pdf

Chile, Consejo Asesor Presidencial para la Educación (2010). Misión. Recuperado de

http://www.consejoeducacion.cl/view/co nsejo-presentacion.asp?opmenu=1\&op-submenu=1

Colegio de Profesores (s.f.). ¿Qué es la evaluación docente? Documento online. Extraído en Octubre 20, 2011 desde Colegio de Profesores. Recuperado de http://www.colegiodeprofesores.cl/index .php?option=com_content\&view=article \&id $=59$ \& temid $=90$

Denzin, N. \& Lincoln, Y. (1998). Estrategias de Investigación Cualitativa. California: Sage Publications.

Duk, C. \& Narvarte, L. (2008). Evaluar la calidad de la respuesta de la escuela a la diversidad de necesidades educativas de los estudiantes. Revista Electrónica Iberoamericana sobre calidad, eficacia y cambio en educación, 6 (002), 137-156. Recuperado de http://redalyc.uaemex.mx/pdf/551/5516 0211.pdf

Elías, S. \& Fernández, M. (2002). Capital Humano y Educación: ¿La calidad importa?. Recuperado de http://www.aaep.org.ar/espa/anales/PD F_02/elias_fernandez.pdf 
Fundación Educación 2020 (2011). ¿Qué es la Prueba INICIA? Área política educativa. Santiago.

Fundación Superación de la Pobreza (2010). Umbrales Sociales para Chile. Hacia una futura política social. Santiago: Autor.

Glaser B. \& Strauss, A. (1967). The Discovering of Grounded Theory: strategies for qualitative research. New York: Aldine.

González, C. (2010). El aprendizaje y el conocimiento académico sobre la enseñanza como clave para mejorar la docencia universitaria. Revista Calidad de la Educación. 33, 124-146.

Jodelet, D. (1986). La representación social: fenómenos, concepto y teoría. En: Moscovici, S. Psicología social II. Barcelona: Paidós,

Moscovici, S. (1961). El Psicoanálisis, su imagen y su público. Buenos Aires: Huemul S.A.

Moscovici, S. (1984). El fenómeno de las representaciones sociales. Cambridge, Inglaterra: Cambridge University Press.

Organization for Economic Co-operation and Development (2009). Tertiary Education in Chile. Paris: OECD and World Bank. Summary in Spanish.

Organization for Economic Co-operation and Development (2010). Education at a Glance 2010: OECD Indicators. Summary in Spanish.

Organización para las Naciones Unidas para la Educación, la Ciencia y la Cultura (2005). Informe Mundial de la UNESCO: Hacia las Sociedades del Conocimiento. Extraído en Abril 20, 2011 desde UNESCO. Acceso: $\mathrm{http} /$ /www.unesco.org/publications

Páez, D., Fernández, I., Ubillos, S. \& Zubieta, E. (2003). Psicología social, cultura y educación. Madrid: Pearson Prentice Hall.

Pavéz, K. (2011, 21 Mayo). Proponen subsecretaría de educación superior y aumento de la subvención preferencial. La Tercera. Recuperado de http://www.latercera.com/noticia/educa cion/2011/05/657-367414-9-proponensubsecretaria-de-educacion-superior-yaumento-de-la-subvencion.shtml

Román, M. (2011, Julio 7). Análisis: educación chilena entre las más costosas. La Nación Mundo. Extraído en Diciembre 29, 2011 desde La Nación Mundo. Recuperado de http://www.nacion.com/2011-0714/Mundo/analisis--educacion-chilenaentre-las-mas-costosas.aspx

Schiefelbein, E., Corvalán, A. \& Peruzzi, S. (2005). Calidad de la Educación, Desarrollo, Equidad y Pobreza en la región, 1980 - 1994. Boletín 38, diciembre 1995. Proyecto Principal de Educación. Recuperado de unesdoc.unesco.org/images/0010/0010 35/103521s.pdf

Trigwell, K. \& Ashwin, P. (2006). An exploratory study of situated conceptions of learning and learning environments. Higher Education, 51(2), pp. 243-25.

United Nations Educational, Scientific and Cultural Organization (2006). SOS Profesión Docente: Al rescate del currículum escolar. IBE Working Papers on Curriculum Issues $\mathrm{N}^{\circ} 2$. Recuperado de http://www.ibe.unesco.org/fileadmin/us er_upload/Publications/Working_Paper s/teachers_latamerica_ibewpci_2.pdf

United Nations Educational, Scientific and Cultural Organization (2007). El derecho a una educación de calidad para todos en Amércia Latina y el Caribe. Revista Iberoamericana sobre calidad, eficacia y cambio en educación. 5(3). Recuperado de http://www.rinace.net/arts/vol5num3/art 1_htm.htm

United Nations Educational, Scientific and Cultural Organization (2010). Datos Mundiales de Educación 2010/11. Recuperado de http://www.ibe.unesco.org/fileadmin/us er_upload/Publications/WDE/2010/pdfversions/Chile.pdf 
Vaillant, D. (2002). Formación de Formadores. Estado de la Práctica. Programa de Promoción de la Reforma Educativa en América Latina y el Caribe. Ed. San Marino: Uruguay. Recuperado de http://todospelaeducacao.org.br/arquivo s/biblioteca/8cc89a87-c5a9-4dc7-9f3a2bb8287687a3.pdf

Valles, M. (2003). Técnicas cualitativas de investigación social. Madrid: Síntesis. 